\title{
Compact and Highly Efficient Grating Couplers Between Optical Fiber and Nanophotonic Waveguides
}

\author{
Frederik Van Laere, Student Member, IEEE, Günther Roelkens, Student Member, IEEE, \\ Melanie Ayre, Jonathan Schrauwen, Student Member, IEEE, Dirk Taillaert, Member, IEEE, \\ Dries Van Thourhout, Member, IEEE, Thomas F. Krauss, and Roel Baets, Senior Member, IEEE, Member, OSA
}

\begin{abstract}
We present high-efficiency grating couplers for coupling between a single-mode fiber and nanophotonic waveguides, fabricated both in silicon-on-insulator (SOI) and InP membranes using BenzoCycloButene wafer bonding. The coupling efficiency is substantially increased by adding a gold bottom mirror to the structures. The measured coupling efficiency to fiber is $69 \%$ for SOI grating couplers and $56 \%$ for bonded InP membrane grating couplers.
\end{abstract}

Index Terms-BenzoCycloButene (BCB) wafer bonding, gold mirror, grating couplers, integrated optics.

\section{INTRODUCTION}

C OUPLING to fiber remains a serious issue in integrated optics. The large difference in dimensions between the fiber and the waveguides on a chip causes high insertion losses and high packaging costs. The ongoing trend to make components smaller, in order to integrate them on one single chip, makes the problem even more difficult. The key features of an effective solution are compactness, low insertion loss, large alignment tolerance, and broadband operation.

Several approaches have been followed to tackle the problem. A very elegant solution is the inverted taper approach, for which low loss and broadband operation were demonstrated in [1]-[3]. These structures however still require lensed or high numerical aperture fiber for a reduced optical mode size.

We use grating couplers for out-of-plane coupling between a standard single-mode fiber and a waveguide. The principle is shown in Fig. 1.

This approach has some major advantages over edgecoupling methods. There is no need for a cleaved facet, and light can be coupled in and out everywhere on the chip, opening the way for wafer-scale testing. Traditional grating couplers

Manuscript received June 29, 2006. This work was supported in part by the European Union under the Information Society Technologies (IST) project FUNFOX and the IST Network of Excellence ePIXnet. The authors would like to thank Alcatel-Thales III-V laboratory for providing the InP-epiwafers.

F. Van Laere, G. Roelkens, J. Schrauwen, D. Taillaert, D. Van Thourhout, and R. Baets are with the Department of Information Technology (INTEC), Ghent University-Interuniversity Microelectronics Center (IMEC), 9000 Ghent, Belgium (e-mail: frederik.vanlaere@ intec.ugent.be).

M. Ayre and T. F. Krauss are with the School of Physics and Astronomy, University of St. Andrews, KY16 9SS St. Andrews, U.K.

Color versions of one or more of the figures in this paper are available online at http://ieeexplore.ieee.org.

Digital Object Identifier 10.1109/JLT.2006.888164

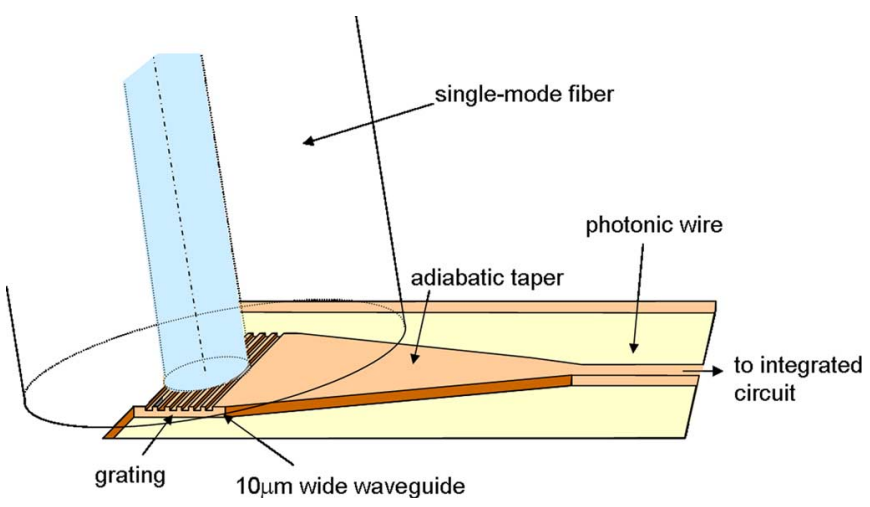

Fig. 1. Coupling principle between fiber and photonic wires by means of a grating.

have a small coupling strength and are therefore rather long and have a small bandwidth. A coupling efficiency of $40 \%$ was reported in [4]. By using a top mirror and coupling through the substrate, a coupling efficiency of 57\% was demonstrated in [5]. In both cases, an additional lens is needed to couple to the fiber. We use a strong and compact grating, resulting in a relatively large bandwidth. A coupling efficiency to single-mode fiber of $33 \%$ for a $10 \times 10-\mu \mathrm{m}^{2}$ grating coupler in silicon-oninsulator (SOI) was demonstrated in [6]. The coupler has a 1-dB bandwidth of $40 \mathrm{~nm}$ and shows good alignment tolerances ( $\pm 2 \mu \mathrm{m}$ for $1-\mathrm{dB}$ excess loss). A 2-D-grating version can be used for getting polarization independence through polarization diversity [7].

For integration with active optoelectronic components, it would be interesting to implement these couplers also in III-V material. For telecom applications, $\mathrm{InP} / \mathrm{InGaAsP}$ is the material system of interest. However, it is impossible to easily transfer existing designs for SOI gratings to InP. The vertical index contrast of InP-based heterostructures is too modest for this purpose. In [8], we modified the vertical index contrast by using BenzoCycloButene (BCB) wafer bonding. We demonstrated grating couplers in bonded InP membranes with a coupling efficiency of $30 \%$.

For both SOI and InP membrane couplers, the coupling efficiency is limited by radiation toward the substrate. In this paper, we solve this problem by adding a gold bottom mirror to grating couplers in SOI and bonded InP membranes. The measured coupling efficiencies are $69 \%$ and $56 \%$, respectively. 


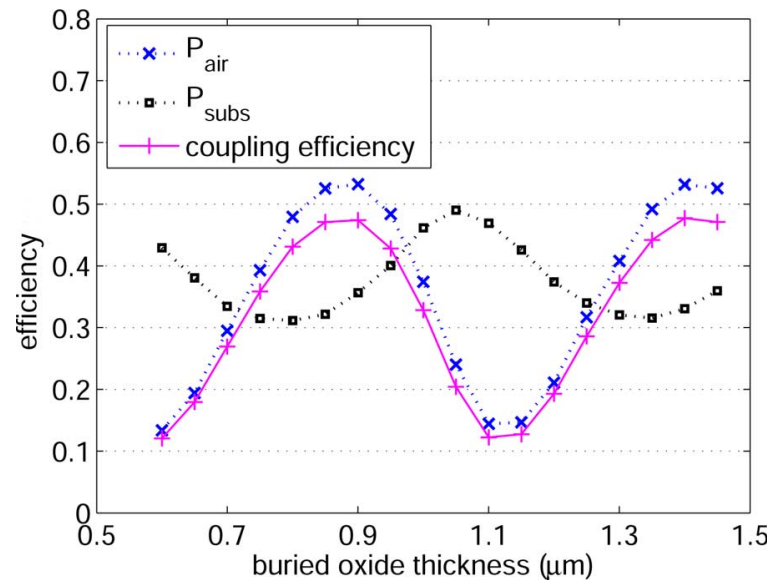

Fig. 2. Upward radiated power, downward radiated power, and coupling efficiency of a regular SOI grating coupler.

\section{GRATING COUPLERS IN SOI}

\section{A. Design}

1) Starting Point: We start from an existing SOI grating coupler ( $\mathrm{Si}$ core layer of $220 \mathrm{~nm}$ and $\mathrm{SiO}_{2}$ buried oxide layer of $1 \mu \mathrm{m})$. The gratings were designed for TE polarization, using CAvity Modelling FRamework (CAMFR, an eigenmode expansion tool) [9]. The design method is described in detail in [10]. We use near vertical coupling (at $10^{\circ}$ with respect to the vertical axis) to avoid second-order reflection and consider coupling from waveguide to fiber (coupling from fiber to waveguide is the same, since we consider coupling between two modes).

The thickness of the buried oxide has a major influence on the coupling efficiency, as described in [11] and [12]. The downward radiated wave partially reflects at the oxide-substrate interface. The oxide thickness should be chosen such that the reflected wave interferes constructively with the directly upward radiated wave. For the used grating (period $=610 \mathrm{~nm}$, etch depth $=50 \mathrm{~nm}$, duty cycle $=50 \%, 20$ periods), the power coupled upward $\left(P_{\text {air }}\right)$, the power coupled toward the substrate $\left(P_{\text {subs }}\right)$, and the coupling efficiency to fiber are shown as a function of the buried oxide thickness in Fig. 2 for the central wavelength of the coupler.

Some care should be taken when discussing the efficiency values. In grating literature, coupling efficiency is often defined as the fraction of the power that is coupled in or out by the grating [13]. Here, we calculate the coupling efficiency to fiber by multiplying $P_{\text {air }}$ by the overlap integral of the outcoupled field profile and the fiber mode. For the used SOI structure, the oxide layer thickness is fixed to $1 \mu \mathrm{m}$, while the optimal thickness is $900 \mathrm{~nm}$. The grating coupler has a theoretical coupling efficiency to fiber of $30 \%$. An important part of the power $(45 \%)$ is radiated into the substrate, since the oxide-substrate interface is not a perfect reflector. A field plot of the structure is shown in Fig. 3.

2) Extension With a Gold Bottom Mirror: The coupling efficiency can be increased substantially by using a nearly perfect reflector as a bottom mirror. We have extended the SOI grating coupler with a gold bottom mirror. Before applying the gold

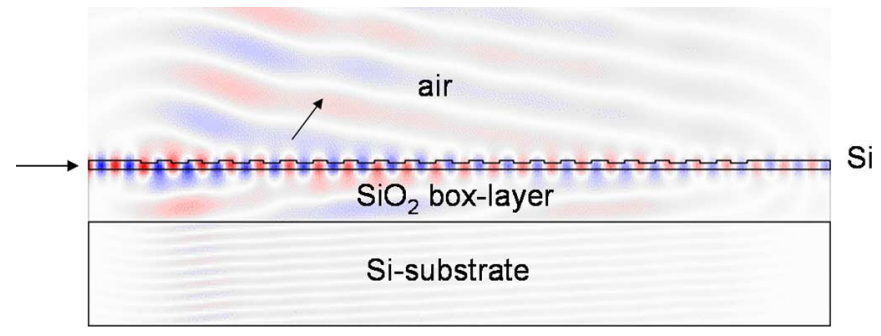

Fig. 3. Field plot of the SOI grating coupler. Forty-five percent of the power is radiated toward the substrate.

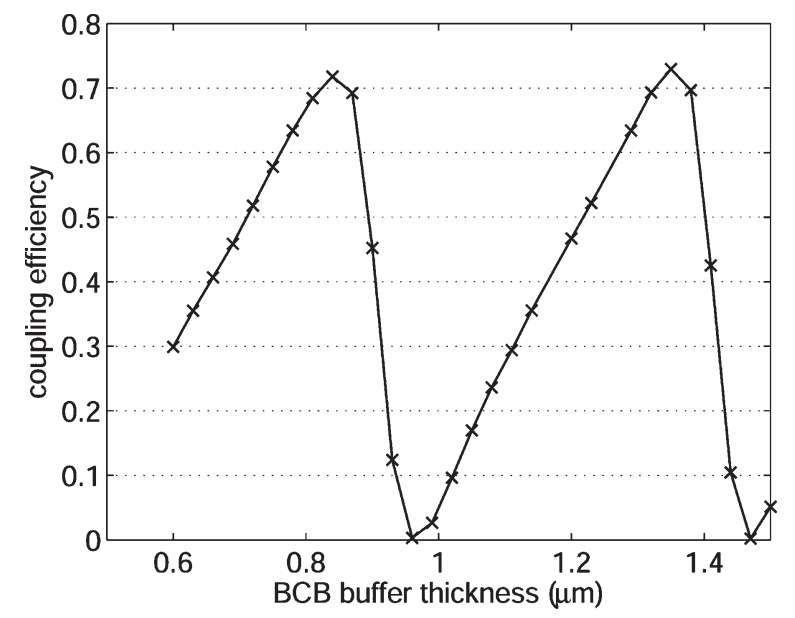

Fig. 4. Determination of the optimal BCB buffer thickness for the SOI coupler with a gold bottom mirror.

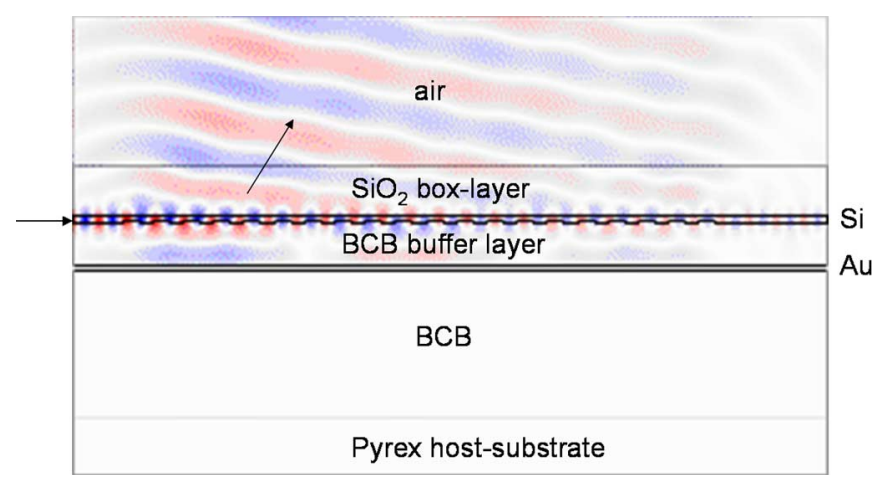

Fig. 5. Field plot of the optimized SOI structure with a gold bottom mirror.

mirror, a low-index buffer layer has to cover the grating. We chose BCB-a low-index ( $n=1.54$ at $\lambda=1.55 \mu \mathrm{m})$ polymer with good planarization properties - as the buffer layer. Its thickness is optimized in order to get the constructive interference between the directly upward radiated wave and the reflected wave at the bottom mirror. The optimal simulated BCB buffer thickness is $840 \mathrm{~nm}$, as shown in Fig. 4 .

The SOI structure, with buffer layer and gold mirror, is bonded onto a host substrate (pyrex in our case) with another $\mathrm{BCB}$ layer (of which the thickness is irrelevant for device efficiency), and the Si substrate is removed. The $1-\mu \mathrm{m}$ buried oxide layer remains as the top layer. The theoretical coupling efficiency to fiber is $72 \%$. A field plot of this structure is shown in Fig. 5. No power is radiated toward the substrate anymore. 


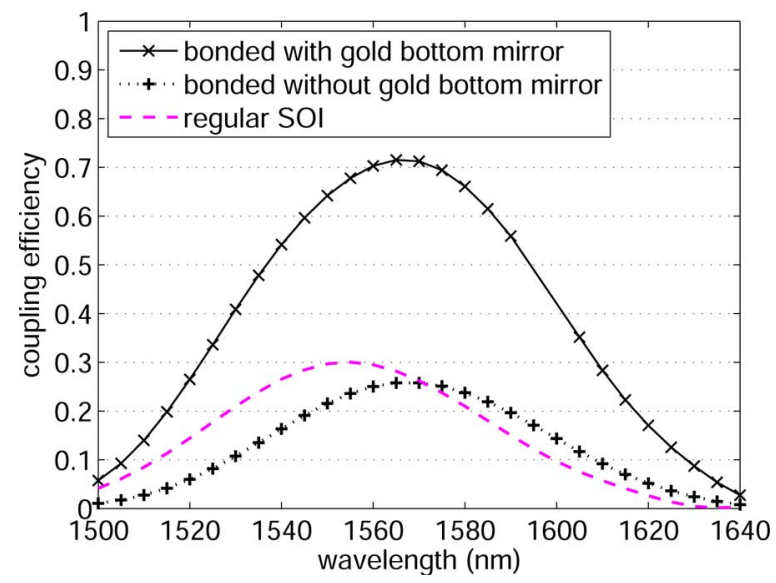

Fig. 6. Simulation results for the different types of SOI grating couplers.
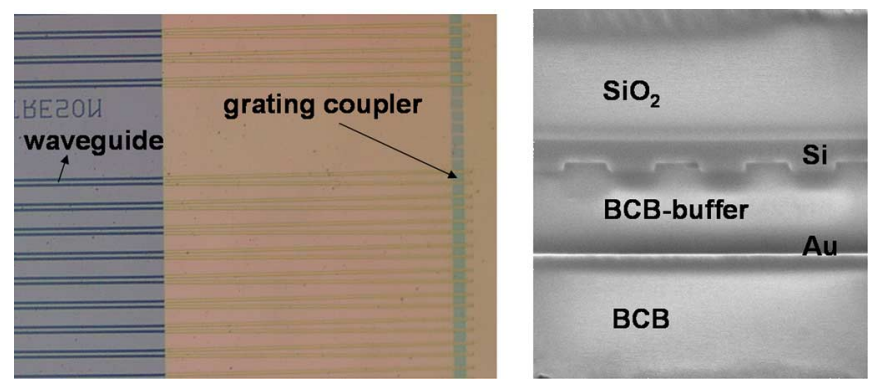

Fig. 7. (Left) Top view of the fabricated SOI structure with a bottom mirror. (Right) SEM picture of an FIB cross section.

The simulations are summarized in Fig. 6. The coupling curves of an SOI grating coupler (1- $\mu \mathrm{m}$ buried-oxide-layer thickness), a bonded SOI grating coupler (without a bottom mirror), and a bonded SOI grating coupler with a bottom mirror are shown.

\section{B. Fabrication}

The SOI structures were defined using $248 \mathrm{~nm}$ deep ultraviolet (DUV) lithography and were etched to a depth of $50 \mathrm{~nm}$ with reactive ion etching (RIE). More details can be found in [14]. After cleaning the samples, a BCB layer of $840 \mathrm{~nm}$ was spin coated onto the SOI die and cured for $40 \mathrm{~min}$ at $210{ }^{\circ} \mathrm{C}$. Afterwards, only the grating area was covered with a $50 \mathrm{~nm}$ evaporated gold layer using lift-off (Fig. 7). The die was then bonded under vacuum onto a pyrex substrate using an approximately $3-\mu \mathrm{m}$ thick BCB layer. After curing the $\mathrm{BCB}$ for $1 \mathrm{~h}$ at $250{ }^{\circ} \mathrm{C}$, the silicon substrate was removed using lapping, $\mathrm{SF}_{6}$ plasma etching, and wet etching in $\mathrm{KOH}$ using the SOI buried oxide layer as an etch-stop layer. A picture of the fabricated structure is shown in Fig. 7 (left), together with an SEM picture of a focused ion beam (FIB) cross section (right).

\section{Measurements}

The structures consist of an input grating coupler and an output coupler connected by a waveguide $(10-\mu \mathrm{m}$ wide tapered to a width of $3 \mu \mathrm{m}$ ). Only the input coupler has a gold bottom mirror. On the sample, we also have bonded reference structures

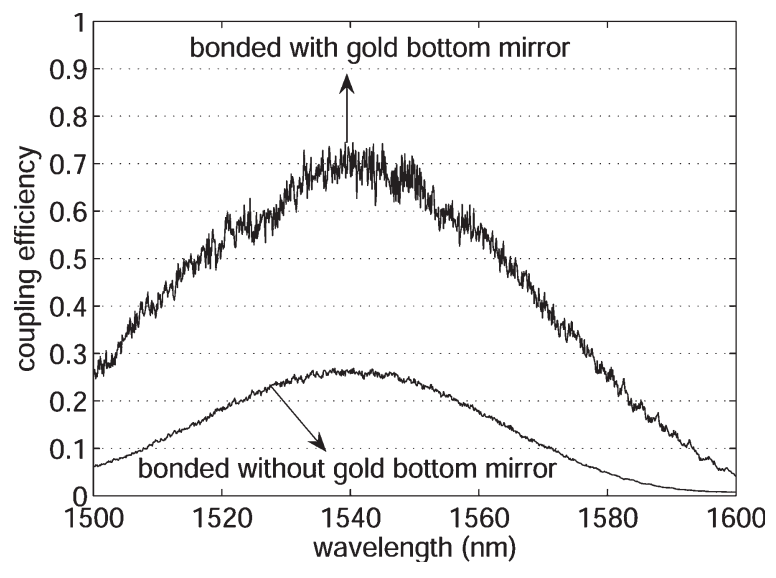

Fig. 8. Measurement results for bonded SOI couplers with and without a gold bottom mirror.

without a bottom mirror, allowing us to evaluate the performance of the mirror. The coupling efficiency is determined from a fiber-to-fiber transmission measurement for the TE polarization. A fiber connected to a tunable laser is positioned at $10^{\circ}$ with respect to the vertical axis above the input grating. Another fiber is positioned above the output grating (also at $10^{\circ}$ ) and connected to a power detector. The position of the fibers is optimized for maximum transmission. By neglecting the waveguide losses (since the waveguides are $3-10-\mu \mathrm{m}$ wide) and by measuring the transmission efficiency of our setup, we are able to calculate the coupling efficiency.

First, the coupling efficiency of a bonded grating coupler without a bottom mirror is determined by measuring the transmission of the reference structures (no mirrors). By assuming that input and output couplers are the same, the coupling efficiency is calculated to be $26 \%$. This value can be used to determine the coupling efficiency of a grating coupler with a bottom mirror. We have measured $69 \%$ coupling efficiency for a grating coupler with a bottom mirror. The measurement results are shown in Fig. 8. The coupling efficiency is deduced from the best fit Gaussian function.

\section{GRating Couplers in InP Membrane}

\section{A. Rationale}

SOI is very well suited for performing passive functionality, but it is very difficult to make active components in SOI. Heterogeneous integration of III-V material (active functions) and SOI (passive functions) could be a viable solution [15].

Another possibility is to perform both active and passive functions in InP/InGaAsP. However, the vertical index contrast of a classical InP/InGaAsP heterostructure is too low for making waveguides with a strong vertical confinement, as is the case in SOI. By applying wafer bonding, the vertical index contrast can be modified, resulting in an InP membrane with high vertical index contrast. These membrane components can then be integrated with classical InP heterostructure components. 


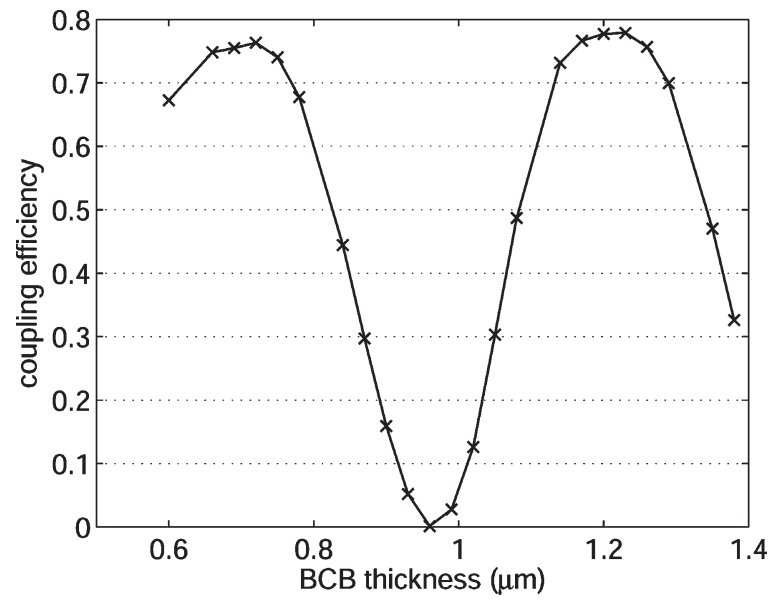

Fig. 9. Determination of the optimal BCB thickness for an InP membrane coupler with a gold bottom mirror.

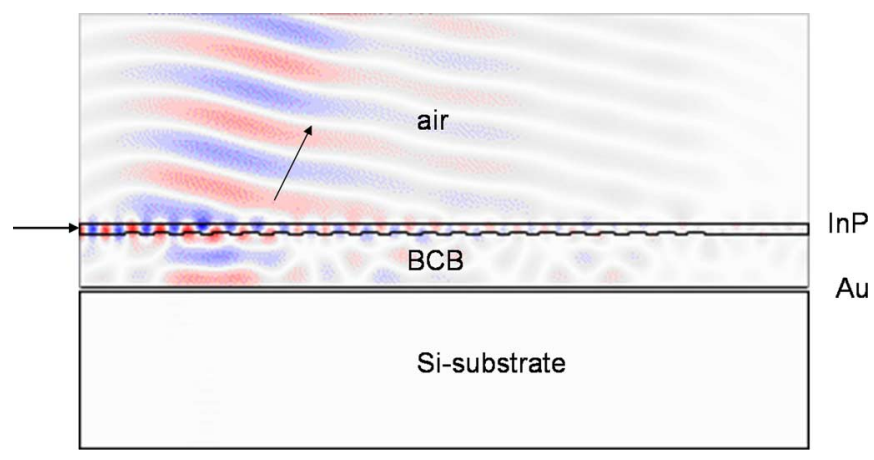

Fig. 10. Field plot of the optimal InP membrane structure.

\section{B. Design}

The design method is similar to the method for SOI grating couplers. Again, a gold bottom mirror is added to the structure to avoid radiation toward the substrate. The InP membrane has a thickness of $300 \mathrm{~nm}$. The period of the optimized grating is $660 \mathrm{~nm}$, the etch depth is $70 \mathrm{~nm}$, and the duty cycle is $50 \%$. We bond onto a gold-coated substrate, meaning that the bonding layer serves also as the buffer layer. The thickness of this BCB layer is again optimized in order to get a constructive interference between the reflected wave at the gold mirror and the directly upward radiated wave. The maximum coupling efficiency as a function of BCB thickness (for the central wavelength of the coupler) is shown in Fig. 9. The optimal thickness is $1.23 \mu \mathrm{m}$, and the maximum coupling efficiency is $78 \%$. A field plot of the optimal structure is shown in Fig. 10.

\section{Fabrication}

The layer structure used for the devices consists of an InP substrate, an InGaAsP etch-stop layer, and a 300-nm InP layer, serving as the membrane layer. In the first step, gratings and waveguides are defined by e-beam lithography using polymethyl methacrylate. The waveguides are $12-\mu \mathrm{m}$ wide and bounded by $3-\mu \mathrm{m}$ wide trenches. The pattern is then transferred into plasma-enhanced chemical vapor deposition (PECVD)deposited $\mathrm{SiO}_{2}$ hard mask by RIE using $\mathrm{CHF}_{3}$. Finally,

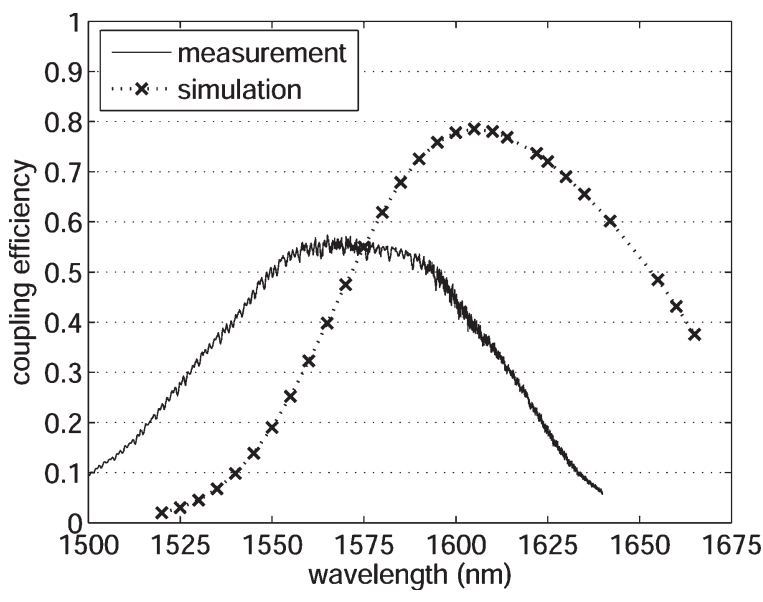

Fig. 11. Measured and simulated coupling efficiency of bonded InP membrane grating couplers with a gold bottom mirror.

the structures are etched into the epistructure by inductively coupled plasma (ICP) using $\mathrm{CH}_{4} / \mathrm{H}_{2}$, and the hard mask layer is removed with HF. The InP samples are much smaller than the SOI samples described above. It is very difficult to spin a uniform BCB buffer layer on such small samples. Therefore, we bond the samples onto a gold-coated $\mathrm{Si}$ substrate by means of $\mathrm{BCB}$, with the grating at the bottom side. The BCB thickness is targeted at $1.23 \mu \mathrm{m}$. After curing the $\mathrm{BCB}$ for $1 \mathrm{~h}$ at $250{ }^{\circ} \mathrm{C}$ in a nitrogen environment, the InP substrate is removed by lapping and wet etching in $\mathrm{HCl}$. Finally, the etch-stop layer is removed by wet etching in $\mathrm{H}_{2} \mathrm{O}: \mathrm{H}_{2} \mathrm{SO}_{4}: \mathrm{H}_{2} \mathrm{O}_{2}$.

\section{Measurements}

The coupling efficiency is determined from a fiber-to-fiber transmission measurement. In this case, both input and output couplers have bottom mirrors. By assuming that input and output couplers are the same, we calculate the coupling efficiency of a single coupler from the transmission efficiency. The measurement result is shown in Fig. 11, together with the simulated curve. The measured coupling efficiency is $56 \%$, and the $1-\mathrm{dB}$ bandwidth is around $45 \mathrm{~nm}$. The deviation between theory and experiment is caused by a deviation between theoretical and fabricated structure (etch depth, filling factor, and $\mathrm{BCB}$ thickness).

\section{CONCLUSION}

We have designed and fabricated highly efficient grating couplers for coupling between a single-mode fiber and nanophotonic waveguides in SOI and bonded InP membranes. The addition of a gold bottom mirror results in high coupling efficiency values. We have measured $69 \%$ coupling efficiency for SOI grating couplers and $56 \%$ for grating couplers in an InP membrane. As these couplers are very compact and have low insertion losses and relatively broadband operation, they meet the requirements for application in future telecommunication networks. Further improvements include the apodization of the grating (varying groove widths and etch depths), which would increase the theoretical coupling efficiency to over $90 \%$. 


\section{ACKNOWLEDGMENT}

F. Van Laere and D. Taillaert would like to thank the Institute for the Promotion of Innovation through Science and Technology (IWT Flanders) for a scholarship and a postdoctoral grant, respectively. Part of this work was performed in the framework of the Institute for the Promotion of Innovation through Science and Technology in Flanders-Strategic Base Research (IWT-SBO) epSOC project, the Institute for the Promotion of Innovation through Science and Technology in Flanders-Generic Base Research at the Universities (IWTGBOU) project on Plastic Photonics, and the Interuniversity Attraction Pole (IAP) Photon Network. The work of G. Roelkens was supported by the Fund for Scientific Research (FWO).

\section{REFERENCES}

[1] T. Shoji, T. Tsuchizawa, T. Watanabe, K. Yamada, and H. Morita, "Low loss mode size converter from $0.3 \mu \mathrm{m}$ square $\mathrm{Si}$ wire waveguides to singlemode fibres," Electron. Lett., vol. 38, no. 25, pp. 1669-1670, Dec. 2002.

[2] S. J. McNab, N. Moll, and Y. A. Vlasov, "Ultra-low loss photonic integrated circuit with membrane-type photonic crystal waveguides," Opt. Express, vol. 11, no. 22, pp. 2927-2939, Nov. 2003.

[3] G. Roelkens, P. Dumon, W. Bogaerts, D. Van Thourhout, and R. Baets, "Efficient silicon-on-insulator fiber coupler fabricated using $248-\mathrm{nm}$ deep UV lithography," IEEE Photon. Technol. Lett., vol. 17, no. 12, pp. 2613-2615, Dec. 2005.

[4] S. Lardenois, D. Pascal, L. Vivien, E. Cassan, S. Laval, R. Orobtchouk, M. Heitzmann, N. Bouzaida, and L. Mollard, "Low-loss submicrometer silicon-on-insulator rib waveguides and corner mirrors," Opt. Lett., vol. 28, no. 13, pp. 1150-1152, Jul. 2003.

[5] R. Orobtchouk, A. Layadi, H. Gualous, D. Pascal, A. Koster, and S. Laval, "High-efficiency light coupling in a submicrometric siliconon-insulator waveguide," Appl. Opt., vol. 39, no. 31, pp. 5773-5777, Nov. 2000.

[6] D. Taillaert, R. Baets, P. Dumon, W. Bogaerts, D. Van Thourhout, B. Luyssaert, V. Wiaux, S. Beckx, and J. Wouters, "Silicon-on-insulator platform for integrated wavelength-selective components," in Proc. IEEE/LEOS Workshop Fibres and Opt. Passive, Italy, 2005, pp. 115-120.

[7] D. Taillaert, H. Chong, P. Borel, L. Frandsen, R. M. De La Rue, and R. Baets, "A compact two-dimensional grating coupler used as a polarisation splitter," IEEE Photon. Technol. Lett., vol. 15, no. 9, pp. 1249-1251, Sep. 2003.

[8] F. Van Laere, M. Ayre, D. Taillaert, D. Van Thourhout, T. F. Krauss, and R. Baets, "Compact and efficient fibre-to-waveguide grating couplers in InP membrane," Electron. Lett., vol. 42, no. 6, pp. 343-345, Mar. 2006.

[9] P. Bienstman and R. Baets, "Optical modelling of photonic crystals and VCSELs using eigenmode expansion and perfectly matched layers," Opt. Quantum Electron., vol. 33, no. 4/5, pp. 327-341, Apr. 2001.

[10] D. Taillaert, P. Bienstman, and R. Baets, "Compact efficient broadband grating coupler for silicon-on-insulator waveguides," Opt. Lett., vol. 29, no. 23, pp. 2749-2751, Dec. 2004.

[11] T. Suhara and H. Nishihara, "Integrated-optics components and devices using periodic structures," IEEE J. Quantum Electron., vol. QE-22, no. 6, pp. 845-867, Jun. 1986.

[12] R. M. Emmons and D. G. Hall, "Buried-oxide silicon-on-insulator structures. 2. Wave-guide grating couplers," IEEE J. Quantum Electron., vol. 28, no. 1, pp. 164-175, Jan. 1992.

[13] T. W. Ang, G. T. Reed, A. Vonsovici, A. G. R. Evans, P. R. Routley, and M. R. Josey, "Effects of grating heights on highly efficient unibond SOI waveguide grating couplers," IEEE Photon. Technol. Lett., vol. 12, no. 1, pp. 59-61, Jan. 2000.

[14] W. Bogaerts, R. Baets, P. Dumon, V. Wiaux, S. Beckx, D. Taillaert, B. Luyssaert, J. Van Campenhout, P. Bienstman, and D. Van Thourhout, "Nanophotonic waveguides in silicon-on-insulator fabricated with CMOS technology," J. Lightw. Technol., vol. 23, no. 1, pp. 401-412, Jan. 2005.

[15] H. T. Hattori, C. Seassal, E. Touraille, P. Rojo-Rmeo, X. Letartre, G. Hollinger, P. Viktorovitch, L. Di Cioccio, M. Zussy, L. El Melhaoui, and J. M. Fedeli, "Heterogeneous integration of microdisk lasers on silicon strip waveguides for optical interconnects," IEEE Photon. Technol. Lett., vol. 18, no. 1, pp. 223-225, Jan. 2006.

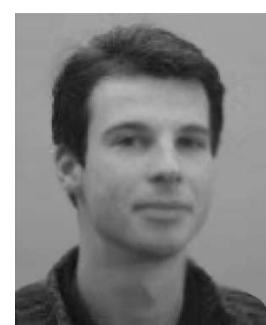

Frederik Van Laere (S'05) received the degree in electrical engineering from Ghent University, Ghent, Belgium, in 2004, where he is currently working toward the $\mathrm{Ph} . \mathrm{D}$. degree in electrical engineering at the Department of Information Technology.

$\mathrm{He}$ is currently working on the design, fabrication, and characterization of nanophotonic waveguide components and their integration with active functionality. $\mathrm{He}$ is currently with the Department of Information Technology, Ghent UniversityInteruniversity Microelectronics Center (IMEC).

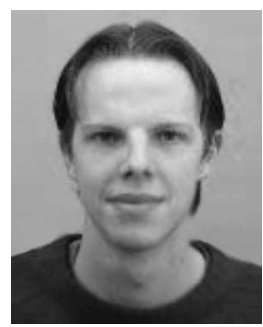

Günther Roelkens (S'02) received the electronic engineering degree from Ghent University, Ghent, Belgium, in 2002

Since 2002, he has been with the Department of Information Technology, Ghent UniversityInteruniversity Microelectronics Center (IMEC). He is currently working in the field of heterogeneous integration.

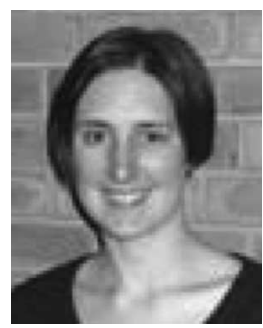

Melanie Ayre received the first class M.Eng. (Hons) degree from University of Glasgow, Glasgow, U.K., in 2002. She is currently working toward the Ph.D. degree in the Microphotonics and Photonic Crystals group with the School of Physics and Astronomy, University of St. Andrews, St. Andrews, U.K., focusing on the design, simulation, fabrication, and characterization of photonic crystal-based devices.

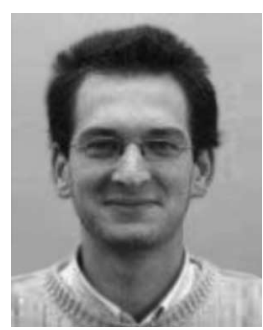

Jonathan Schrauwen (S'05) received the degree in physical engineering from Ghent University, Ghent, Belgium, in 2004, where he is currently working toward the Ph.D. degree.

In 2004, he started working as a Researcher with the Photonics Research Group, Department of Information Technology, Ghent UniversityInteruniversity Microelectronics Center (IMEC). His research area is applications of focused ion beam in photonics.

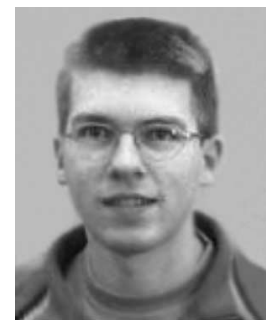

Dirk Taillaert (S'97-M'04) received the M.A. degree in electrical engineering in 1999 and the Ph.D. degree in November 2004, both from Ghent University, Ghent, Belgium.

He started working as a Researcher with the Department of Information Technology, Ghent University-Interuniversity Microelectronics Center (IMEC), in August 1999. Currently, he is working on optical sensors with a postdoctoral grant from the Institute for the Promotion of Innovation through Science and Technology (in Flanders). His research interests include the design, fabrication, and characterization of wavelengthscale components for photonic integrated circuits. 


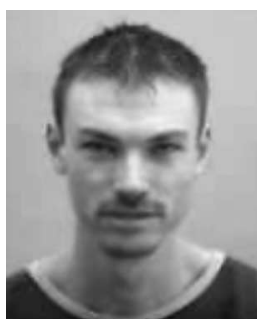

Dries Van Thourhout (M'98) received the physical engineering and the Ph.D. degrees from Ghent University, Ghent, Belgium, in 1995 and 2000, respectively.

He was with the Lucent Technologies, Bell Laboratories, Crawford Hill, NJ, from October 2000 to September 2002, working on the design, processing, and characterization of InP/InGaAsP monolithically integrated devices. In October 2002, he joined the Department of Information Technology, Ghent University-Interuniversity Microelectronics Center, continuing his work on integrated opto-electronic devices. His main research interests are heterogeneous integration by wafer bonding, intrachip optical interconnect, and wavelength-division-multiplexing devices.

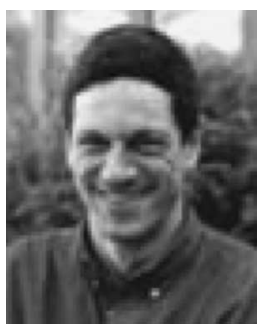

Thomas F. Krauss received the Diplom-Ingenieur degree in photographic engineering from Fachhochschule Köln, Cologne, Germany, in 1989, with a diploma thesis conducted at IBM Research, Yorktown Heights, NY, and the Ph.D. degree in electrical engineering from the University of Glasgow, Glasgow, U.K., in 1992.

During 1993-2000, he held an EPSRC and then a Royal Society Research Fellowship, both at the University of Glasgow, before moving to a Personal Chair in Optoelectronics at the University of St. Andrews, St. Andrews, U.K., in 2000. He is recognized as one of the leading researchers in the field of photonic crystals in Europe and worldwide. He started his photonic-crystal activity with Prof. De La Rue at the University of Glasgow in 1993 and was the first to demonstrate a 2-D photonic bandgap in a semiconductor waveguide structure in 1996. He leads and contributes to a range of projects both at national and European levels, most notably the European Union-Information Society Technologies (EU-IST) Photonic Integrated Circuits using Crystal Optics (PICCO) project that developed photonic circuits based on photonic crystals. He presents invited talks and lectures at international meetings at a rate of 8-12 per year. He contributes strategically to the advancement of the field, e.g., via symposia at the European Conference on Optical Communication, Integrated Photonics Research, Materials Research Society, and Photonic and Electromagnetic Crystal Structure, and was Chairman of the ePIXnet winter school on photonic integration held at Pontresina, Switzerland, in March 2006. He is currently with the School of Physics and Astronomy, University of St. Andrews.

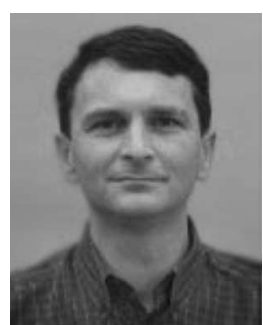

Roel Baets (M'88-SM'96) received the electrical engineering degree from Ghent University, Ghent, Belgium, in 1980, the M.Sc. degree in electrical engineering from Stanford University, Stanford, CA, in 1981, and the Ph.D. degree from Ghent University in 1984.

He joined the Department of Information Technology (INTEC), Ghent University, in 1981, and since 1989, he has been a Professor with the engineering faculty. From 1990 to 1994, he was a Part-Time Professor with the Technical University of Delft, Delft, The Netherlands. He has mainly worked in the field of photonic components. With about 300 publications and conference papers as well as about ten patents, he has made contributions to the design and fabrication of III-V semiconductor laser diodes, passive guided-wave devices, photonic integrated circuits, and micro-optic components. He currently leads the Photonics Group at the INTEC, Ghent University, which is an associated laboratory of the Interuniversity Microelectronics Center, working on integrated photonic devices for optical communication, optical interconnect, and optical sensing.

Dr. Baets is a member of the Optical Society of America, the IEEE Lasers and Electro-Optics Society (where he was formerly a Chairman of the Benelux Chapter from 1999 to 2001 and is currently a member of the Board of Governors, the International Society for Optical Engineers), and the Flemish Engineers Association. He has been member of the program committees of the Optical Fiber Communications Conference, the European Conference on Optical Communication, the IEEE Semiconductor Laser Conference, the European Solid-State Device Research Conference, the Conference on Lasers and Electro-Optics, Europe, and the European Conference on Integrated Optics. 